\title{
Evaluation of the RPM Approach for the Simulation of Broadband Combustion Noise
}

\author{
B. Mühlbauer* \\ DLR, German Aerospace Center, 70569 Stuttgart, Germany \\ R. Ewert $\ddagger$ and O. Kornow \\ DLR, German Aerospace Center, 38108 Braunschweig, Germany \\ and \\ B. Noll $\underline{\S}$ \\ DLR, German Aerospace Center, 70569 Stuttgart, Germany \\ DOI: $\underline{10.2514 / 1.45535}$
}

\begin{abstract}
The derivation and validation of a broadband combustion noise model is presented. The random particle-mesh approach for combustion noise is a hybrid computational fluid dynamics/computational aeroacoustics method and relies on the stochastic reconstruction of combustion noise sources in the time domain. The stochastic reconstruction of unsteady sound sources based on statistical turbulence quantities from a reacting Reynolds-averaged NavierStokes simulation is realized with the random particle-mesh method. In the present paper, the modeled combustion noise sources are derived for the use in conjunction with the linearized Euler equations for the computation of the acoustic propagation. Two open, nonpremixed, turbulent jet flames (DLR-A and DLR-B), which differ in their fuel outlet velocity and their respective Reynolds number, are used for the validation of the particle-mesh for combustion noise approach. Results of the reacting flow computations and the subsequent acoustic simulations are compared with measurements. Excellent agreement is found between the computed narrow band sound spectra and the experimental data.
\end{abstract}

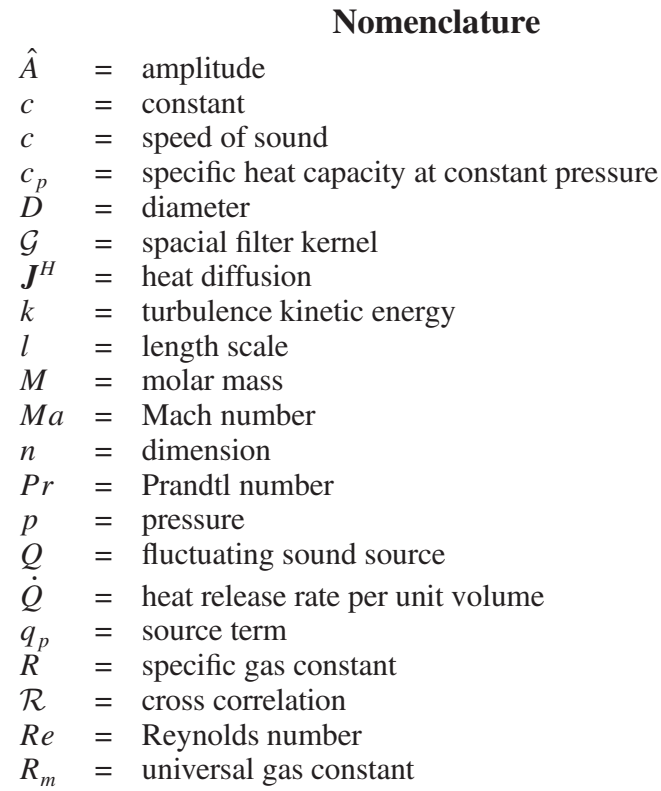

Presented as Paper 3285 at the 15th AIAA/CEAS Aeroacoustics Conference (30th AIAA Aeroacoustics Conference), Miami Florida, 11-13 May 2009; received 2 June 2009; revision received 17 March 2010; accepted for publication 27 March 2010. Copyright (C) 2010 by German Aerospace Center. Published by the American Institute of Aeronautics and Astronautics, Inc., with permission. Copies of this paper may be made for personal or internal use, on condition that the copier pay the $\$ 10.00$ per-copy fee to the Copyright Clearance Center, Inc., 222 Rosewood Drive, Danvers, MA 01923; include the code 0001-1452/10 and \$10.00 in correspondence with the CCC.

${ }^{*}$ Research Associate, Institute of Combustion Technology, Computer Simulation. Member AIAA.

Research Engineer, Institute of Aerodynamics and Flow Technology, Technical Acoustics. Member AIAA.

Research Associate, Institute of Aerodynamics and Flow Technology, Technical Acoustics.

${ }^{\S}$ Research Engineer, Institute of Combustion Technology, Computer Simulation. Member AIAA.

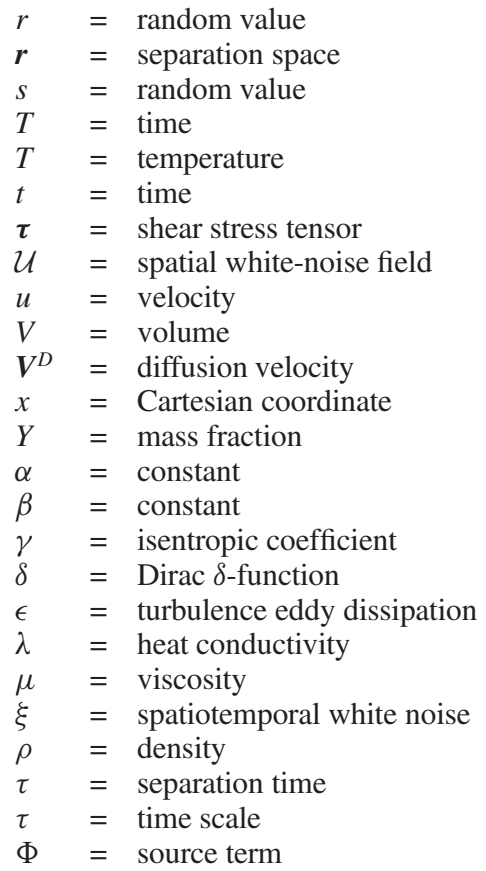

\section{Introduction}

C OMBUSTION chambers in modern aeroengines are operated under lean premixed conditions and chemical reactions occur in highly turbulent recirculation flows. Because of the unsteadiness of turbulent combustion, broadband combustion noise is generated. The numerical simulation of broadband combustion noise is a complex task because turbulence, chemical reactions, and acoustics have to be resolved simultaneously. The vastly different scales of the dynamics of turbulence, chemical reactions, and acoustics as well as the need for low-dissipation and low-dispersion high-order discretization schemes triggered the development of hybrid computational fluid dynamics/computational aeroacoustics (CFD/CAA) approaches in previous investigations, where the combustion noise 
problem was split into a turbulent reacting flow part to be treated with CFD and a subsequent aeroacoustic simulation by means of CAA methods.

Currently, hybrid approaches for the numerical simulation of combustion noise are applied primarily based on acoustic sources computed by large eddy simulation (LES). Subsequently, the acoustic propagation is predicted by solving the acoustic perturbation equations for reacting flows (APE-RF) [1] or wave equations [2,3]. For sound propagation over larger distances, however, $\mathrm{C} \overline{\mathrm{A}} \overline{\mathrm{A}}$ methods based on a discretization of the simulation domain with spatial meshes become increasingly expensive. In case of larger propagation distances it is necessary to sample the unsteady acoustic data on a surface that surrounds the flame and extrapolate it with, e.g., analytical methods such as Kirchhoff's surface integral [4] or a porous Ffowcs Williams and Hawkings methods into the far field [5]. Computationally more efficient methods of predicting combustion noise spectra arise from statistical noise theory [6]. These methods are based on a modeled source term to represent the fluctuating heat release $[7,8]$. Until now, however, stochastically reconstructed noise sources for broadband aeroacoustic predictions were restricted to nonreacting flow problems. In particular airframe noise and jet noise problems were studied with hybrid Reynoldsaveraged Navier-Stokes (RANS)/CAA methods based on stochastic source reconstruction [6,9-12].

In the current investigation, the hybrid random particle-mesh approach for combustion noise (RPM-CN), relying on the stochastic reconstruction of combustion noise sources in the time domain, is derived and compared against experimental data. In contrast to LES/ CAA approaches, the reacting flow simulation is based on a RANS simulation of the combustion problem, which lacks a time accurate resolution of turbulence but rather provides statistical information on the time-averaged flow. From the standard two-equation RANS models only a local turbulent time and length scale can be inferred, i.e., only an integrated measure of the generally anisotropic twopoint correlations is provided. Hence, spatiotemporal correlations and thus the shape of turbulence spectra, which are implicitly related to these, have to be appropriately modeled for a further use of the RANS-based turbulence statistics in the final acoustic simulation.

A first stochastic method to generate spectra and correlation functions of turbulence, which is based on random Fourier modes, was introduced by Kraichnan in the early 1970s [9]. A modified version of this approach was applied by Béchara et al. [6] to model noise sources of free turbulent flows. Further improvements of the stochastic noise generation and radiation (SNGR) method were introduced by Bailly et al. [10], Bailly and Juve [11], and Billson et al. [12]. Kalitzin et al. [13] applied the SNGR approach in a first attempt to predict trailing edge noise. In this paper the random particle-mesh (RPM) method, introduced by Ewert and Edmunds [14] and Ewert [15-17] to model vortex sound sources for airframe and jet-noise, is adapted to reconstruct stochastic fluctuating combustion noise sources in the time domain. The main difference between RPM and the random Fourier mode techniques is that the RPM approach focuses on the realization of spatiotemporal correlations, which implicitly specify the related turbulence spectra, while in the random Fourier mode approach wave-numberfrequency spectra are realized, from which the related correlations derive. Note that a correlations-based model directly allows the inclusion of the source convection effect, because Taylor's hypothesis is originally formulated as a local relationship between time and streamwise one-dimensional correlation. On the contrary, Taylor's hypothesis only remains unchanged in Fourier space if the convection velocity is a global property, i.e., constant. Otherwise, the inclusion of a locally varying convection velocity into the Fourier space model appears to be technically complex, especially if the other parameters such as local decorrelation time scales or turbulence intensities shall remain unaffected.

In a previous investigation [18] acoustic perturbation equations (APE-4) [19] were used to simulate the sound radiation problem. In this acoustic approach a right-hand side (RHS) source based on the heat release rate appears, which was approximated through an expression involving Reynolds temperature fluctuations. If the approach is used for statistical noise modeling, some inconsistencies appear. Because reacting flow RANS simulations provide a Favreaveraged temperature variance, which can differ significantly from a Reynolds-averaged temperature variance, it is necessary to formulate the combustion noise source in terms of a Favre fluctuating temperature. Furthermore, for a more general applicability to reacting flow, the effect of nonisomolar combustion also has to be considered in the source term.

In this investigation the first preliminary formulation [18] is revised. A governing equation for fluctuating pressure is derived, which includes RHS sources based on Favre fluctuating temperature and Favre fluctuating mass fractions. Variances of these quantities are provided by reacting flow RANS simulations, hence the statistical modeling of the noise sources becomes feasible. As will be shown for the newly derived sources, in case of isomolar combustion, the fluctuating temperature term represents the most prominent contributor to the noise source. The pressure equation is used in conjunction with the linearized Euler equations (LEE) as acoustic propagation model. For the derivation of a RPM based combustion noise model the statistical jet noise model of Tam and Auriault [20] served as a guiding paradigm. The statistical modeling of the newly derived source formulation will be discussed in detail.

The results from this numerical approach are validated using experimental data for the DLR-A and DLR-B flames [21]. The open, nonpremixed, turbulent jet flames are well suited test cases because velocity, temperature, species, and acoustic experimental data are available.

The paper is organized as follows. Section II.A gives a brief overview about the RPM method and the statistical features generated by it. In Sec, II.B an acoustic source term based on Favreaveraged temperature fluctuations is derived. It is shown that a source based on temperature fluctuations comprises the major contribution to the source of the pressure-density relation. Section III introduces the DLR-A and DLR-B flame and discusses the CFD and CAA computations. In Sec. IV the results of the simulations are compared with experimental data and discussed.

\section{Modeling}

\section{A. RPM method}

The random particle-mesh (RPM) method was introduced by Ewert [14-17] as a stochastic method to generate unsteady turbulent fields with locally prescribed one- and two-point statistics. The RPM method is an Eulerian-Lagrangian stochastic method, which generates a statistically stationary fluctuating sound source $Q(x, t)$ in the Eulerian frame used in CAA methods. The cross-covariance $\mathcal{R}$ generated by the method as applied in this work is Gaussian in space and exponential in time, i.e.

$$
\begin{gathered}
\mathcal{R}(\boldsymbol{x}, \boldsymbol{r}, \tau)=\langle Q(\boldsymbol{x}, t) Q(\boldsymbol{x}+\boldsymbol{r}, t+\tau)\rangle \\
=\hat{R} \exp \left\{-\frac{|\tau|}{\tau_{s}}-\frac{\pi\left(\boldsymbol{r}-\boldsymbol{u}_{c} \tau\right)^{2}}{4 l_{s}^{2}}\right\}
\end{gathered}
$$

where $\langle\cdots\rangle$ denotes the ensemble average. $\hat{R}$ defines the variance of the correlated quantity for vanishing separation space $\boldsymbol{r}$ and time $\tau$. The parameters $l_{s}$ and $\tau_{s}$ denote integral length- and time scales, respectively. Taylor's hypothesis is taken into account by the convection velocity $\boldsymbol{u}_{c}$. For inhomogeneous turbulence $\hat{R}, l_{s}, \tau_{s}$, and $\boldsymbol{u}_{c}$ depend on the position $\boldsymbol{x}$.

The fluctuating quantity $Q(\boldsymbol{x}, t)$ is obtained by spatially filtering a spatial white-noise field $\mathcal{U}$, which is generated with a specific stochastic partial differential equation. The filtering reads

$$
Q(\boldsymbol{x}, t)=\int_{V_{S}^{n}} \hat{A}(\boldsymbol{x}) \mathcal{G}\left(\left|\boldsymbol{x}-\boldsymbol{x}^{\prime}\right|, l_{s}(\boldsymbol{x})\right) \mathcal{U}\left(\boldsymbol{x}^{\prime}, t\right) \mathrm{d}^{n} \boldsymbol{x}^{\prime}
$$

In this expression $\mathcal{G}$ is a Gaussian spatial filter kernel defined by

$$
\mathcal{G}\left(\xi, l_{s}\right)=\exp \left(-\frac{\pi}{2} \frac{\xi^{2}}{l_{s}^{2}}\right)
$$


which yields a Gaussian spatial correlation of $Q$, with integral length scale $l_{s}$. The dimension of the problem is indicated by $n$ and $V_{S}^{n}$ is considered to be the source region. The amplitude function $\hat{A}$ realizes a local target variance of the fluctuating quantity $Q$; its scaling is discussed below. [22]

The spatial white noise field is generated by a Langevin equation

$$
\frac{\mathrm{D}_{0}}{\mathrm{D} t} \mathcal{U}=-\frac{1}{\tau_{s}} \mathcal{U}+\sqrt{\frac{2}{\rho_{0}^{c} \tau_{s}}} \xi(\boldsymbol{x}, t)
$$

formulated in a Lagrangian frame. Here $\mathrm{D}_{0} / \mathrm{D} t=\partial / \partial t+\boldsymbol{u}_{0}^{c} \cdot \nabla$ denotes a substantial time derivative. The steady velocity field $\boldsymbol{u}_{0}^{c}(\boldsymbol{x})$ determines the ultimately achieved convection velocity $\boldsymbol{u}_{c}$. For a constant velocity $\boldsymbol{u}_{0}^{c}$ the convection velocity becomes $\boldsymbol{u}_{c}=\boldsymbol{u}_{0}^{c}$. However, in general, an arbitrary local convection velocity can be achieved. The density $\rho_{0}^{c}$ is defined such that $\nabla \cdot\left(\rho_{0}^{c} \boldsymbol{u}_{0}^{c}\right)=0$ is satisfied. The quantity $\xi(\boldsymbol{x}, t)$ is Gaussian distributed spatiotemporal white noise, i.e., it satisfies

$$
\begin{gathered}
\langle\xi(\boldsymbol{x}, t)\rangle=0 \\
\langle\xi(\boldsymbol{x}, t) \xi(\boldsymbol{x}+\boldsymbol{r}, t+\tau)\rangle=\delta(\tau) \delta(\boldsymbol{r})
\end{gathered}
$$

where $\delta$ denotes the Dirac $\delta$-function.

Based on the definition for $Q$, Eq. (2) its cross-covariance becomes $[\underline{23}]$

$$
\begin{aligned}
& \mathcal{R}(\boldsymbol{x}, \boldsymbol{r}, \tau)=\langle Q(\boldsymbol{x}, t) Q(\boldsymbol{x}+\boldsymbol{r}, t+\tau)\rangle \\
& \quad=\frac{\hat{A}(\boldsymbol{x}) \hat{A}(\boldsymbol{x}+\boldsymbol{r})}{\rho_{0}^{c}(\boldsymbol{x})} l_{s}^{n}(\boldsymbol{x}) \exp \left(-\frac{|\tau|}{\tau_{s}}-\frac{\pi\left|\boldsymbol{r}-\boldsymbol{u}_{0}^{c} \tau\right|^{2}}{4 l_{s}^{2}(\boldsymbol{x})}\right)
\end{aligned}
$$

To achieve the appropriate variance $\mathcal{R}(\boldsymbol{x}, \mathbf{0}, 0)=\hat{R}$ of Eq. (1) the amplitude $\hat{A}$ must be chosen accordingly

$$
\hat{A}(\boldsymbol{x})=\sqrt{\frac{\rho_{0}^{c}(\boldsymbol{x}) \hat{R}(\boldsymbol{x})}{l_{s}^{n}(\boldsymbol{x})}}
$$

To discretize the filtered stochastic partial differential equation, the convecting white noise field is modeled by convecting particles. Random values are associated to the particles, which are Gaussian deviates with a variance proportional to the inverse of the particle density. A bundle of streamlines covers the source domain that has to be resolved. In this paper, the convection field is identified with the time-averaged mean-flow $\tilde{\boldsymbol{u}}$ from RANS. Random particles are seeded at a constant clock rate at the foremost upstream position on each streamline. The particles drift along the streamline until they are finally removed downstream. The spatial filtering is applied sequentially. In the first step the random values are filtered along the streamline. Next, the values are weighted and distributed in direction normal to the streamline onto the CAA mesh.

To discretize the Langevin equation, Eq. (4) the random values carried by each particle are not kept constant but rather change over time according to the discrete equation [24]

$$
r_{i}^{n+1}=\alpha r_{i}^{n}+\beta s_{i}^{n}
$$

In this case $r_{i}^{n+1}$ and $r_{i}^{n}$ denote the random value of a particle at timelevel $n+1$ and $n$, respectively. The quantity $s_{i}^{n}$ is a Gaussian deviate with same variance as $r_{i}$. This procedure results to an exponential decay [12]. The constant $\alpha$ follows by discretizing the Langevin equation, Eq. (4). It is related to the time scale $\tau_{s}$ via

$$
\alpha=1-\frac{\Delta t}{\tau_{s}}
$$

where $\Delta t$ denotes the time-increment between the levels $n+1$ and $n$. To preserve the variance of $r_{i}$ over time, $\beta$ must be chosen as

$$
\beta=\sqrt{\frac{2 \Delta t}{\tau_{s}}}
$$

\section{B. RPM-CN approach}

The statistical jet noise model of Tam and Auriault [20] was used as a guiding paradigm for the derivation of a RPM based combustion noise model. The source term of the Tam and Auriault jet noise model is based on the substantial time derivative $\mathrm{D}_{0} q_{s} / \mathrm{D} t$ of a quantity $q_{s}$, which could be interpreted as the hydrodynamic pressure fluctuations in the jet. The source term is used in conjunction with the LEEs as a governing equation and appears on the RHS of the pressure equation. Stochastical jet noise simulations were realized by modeling the acoustic source term $\mathrm{D}_{0} q_{s} / \mathrm{D} t$ by the RPM method $[17,23,25,26]$. Therefore, a pressure equation for reacting flows is derived with a substantial time derivative on the RHS. This source term is modeled stochastically using the RPM method and the acoustic propagation is computed by solving the derived pressure equation in combination with the continuity and momentum equation of the original LEE.

Next, a pressure equation for reacting flows with a combustion noise source term on the RHS is derived. Subsequently, the statistical source term modeling is described and the system of equations of the RPM-CN approach is presented. Finally the derived broadband combustion noise model is discussed.

\section{Pressure Equation}

The pressure-density relation reads

$$
\frac{1}{c^{2}} \frac{\mathrm{D} p}{\mathrm{D} t}-\frac{\mathrm{D} \rho}{\mathrm{D} t}=\rho \Phi
$$

with the source $\Phi$ and $c^{2}=\gamma p / \rho$. As discussed by Candel et al. [27] the RHS source becomes

$$
\begin{aligned}
\Phi & =\frac{\dot{Q}}{\rho c_{p} T}+M \frac{\mathrm{D}}{\mathrm{D} t}\left(\frac{1}{M}\right)+\frac{1}{\rho c_{p} T}[\nabla \cdot \lambda \nabla T+\boldsymbol{\tau}: \nabla \boldsymbol{u} \\
& \left.-\sum_{k=1}^{N} \rho Y_{k} c_{p k} \boldsymbol{V}_{k}^{D} \cdot \nabla T\right]
\end{aligned}
$$

where $\dot{Q}$ represents the heat release rate per unit volume, $\rho, c_{p}, T, M$, $\lambda, \boldsymbol{\tau}, \boldsymbol{u}, Y_{k}$, and $\boldsymbol{V}_{k}^{D}$ respectively designate the local density, specific heat capacity at constant pressure, temperature, molar mass, thermal conductivity, viscous stress tensor, velocity vector of the mixture and mass fraction and diffusion velocities of species $k$. The conservation equation of energy relates the heat release rate $\dot{Q}$ to temperature $T$, pressure $p$, viscous stresses $\boldsymbol{\tau}$, and heat diffusion $\boldsymbol{J}^{H}$, i.e.

$$
\rho c_{p} \frac{\mathrm{D} T}{\mathrm{D} t}=\dot{Q}+\frac{\mathrm{D} p}{\mathrm{D} t}+\boldsymbol{\tau}: \nabla \boldsymbol{u}-\nabla \cdot \boldsymbol{J}^{H}
$$

In the following, Reynolds decomposition [28] of an arbitrary quantity $\phi=\bar{\phi}+\phi^{\prime}$ and Favre decomposition [29] $\phi=\tilde{\phi}+\phi^{\prime \prime}$ are used, where $\bar{\phi}=\frac{1}{T} \int_{T} \phi \mathrm{d} t$ and $\tilde{\phi}=\overline{\rho \phi} / \bar{\rho}$. Reynolds averages and Reynolds fluctuations are denoted by $\bar{\phi}$ and $\phi^{\prime}$, respectively. Analogously, $\tilde{\phi}$ and $\phi^{\prime \prime}$ define Favre averages and Favre fluctuations.

The derivation of the pressure equation starts by introducing the continuity equation into Eq. (12)

$$
\frac{\mathrm{D} p}{\mathrm{D} t}+\gamma p \nabla \cdot \boldsymbol{u}=\gamma p \Phi
$$

Applying the Reynolds decomposition and neglecting the nonlinear terms on the left-hand side (LHS) yields

$$
\begin{aligned}
& \frac{\partial p^{\prime}}{\partial t}+\overline{\boldsymbol{u}} \cdot \nabla p^{\prime}+\boldsymbol{u}^{\prime} \cdot \nabla \bar{p}+\overline{\boldsymbol{u}} \cdot \nabla \bar{p}+\gamma \bar{p} \nabla \cdot \overline{\boldsymbol{u}}+\gamma \bar{p} \nabla \cdot \boldsymbol{u}^{\prime} \\
& \quad+\gamma p^{\prime} \nabla \cdot \overline{\boldsymbol{u}}=\gamma p \Phi
\end{aligned}
$$


The time average becomes

$$
\bar{u} \cdot \nabla \bar{p}+\gamma \bar{p} \nabla \cdot \overline{\boldsymbol{u}}=\gamma \overline{p \Phi}
$$

Subtraction of the time average yields

$$
\frac{\partial p^{\prime}}{\partial t}+\overline{\boldsymbol{u}} \cdot \nabla p^{\prime}+\boldsymbol{u}^{\prime} \cdot \nabla \bar{p}+\gamma \bar{p} \nabla \cdot \boldsymbol{u}^{\prime}+\gamma p^{\prime} \nabla \cdot \overline{\boldsymbol{u}}=q_{p}
$$

The LHS is the linearized Euler pressure equation. With $\gamma p / \rho=c^{2}$ the RHS source is given by

$$
q_{p}=(\gamma p \Phi)^{\prime}=\rho c^{2} \Phi-\overline{\rho c^{2} \Phi}
$$

Next, Eq. (14) is used to express the heat release in Eq. (13) as a function of temperature. Then the complete source $\Phi$ of the pressuredensity relation becomes

$$
\Phi=\frac{1}{T} \frac{\mathrm{D} T}{\mathrm{D} t}-\frac{1}{\rho c_{p} T} \frac{\mathrm{D} p}{\mathrm{D} t}+M \frac{\mathrm{D}}{\mathrm{D} t}\left(\frac{1}{M}\right)
$$

In this formulation all viscous and heat convection terms disappear. The three terms of Eq. (20) represent a concise formulation of the full source as given by Eq. (13). This very compact source description follows also directly by expressing the temperature differential as a total derivative

$$
\mathrm{d} T=\left(\frac{\partial T}{\partial p}\right)_{\rho, Y_{k}} \mathrm{~d} p+\left(\frac{\partial T}{\partial \rho}\right)_{p, Y_{k}} \mathrm{~d} \rho+\sum_{k=1}^{N}\left(\frac{\partial T}{\partial Y_{k}}\right)_{\rho, p, Y_{m \neq k}} \mathrm{~d} Y_{k}
$$

For a perfect gas, this expression becomes

$$
\mathrm{d} T=\frac{T}{p} \mathrm{~d} p-\frac{T}{\rho} \mathrm{d} \rho-T \sum_{k=1}^{N} \frac{M}{M_{k}} \mathrm{~d} Y_{k}
$$

from which Eq. (20) as the RHS of the pressure density relation Eq. (12) can be derived directly.

The first term of Eq. (20) is mainly driven by the substantial time derivative of temperature, the second by the substantial time derivative of pressure. The first term will mainly be effective for hot or reacting jets. The second term is similar to the jet noise source term that is used in the Tam and Auriault model [20]. Therefore, it is mainly attributed to the sound generated by turbulent velocity fluctuations in a cold jet. Because for low Mach number combustion the sound generated by turbulent velocity fluctuations is negligible compared with temperature effects [30], in this case the first term must be dominant over the second. The third term will come into picture if significant differences exist between the average molecular weight of reaction products and reactants for reacting flows. Therefore, in the case of quasi-isomolar reacting flows the first term represents the most significant contribution to the noise source term. That is, the temperature source term is not just one of many contributors to the heat release term, which in turn is just one of many contributors to the pressure-density relations source term. Rather the temperature fluctuations represent for quasi-isomolar combustion the most prominent contributor out of the three terms that describe the pressure-density relation source.

Using Eq. (19) with Eq. (20) the source term of the linearized pressure equation, Eq. (18) becomes

$$
q_{p}=\left(\gamma R_{m} \rho \frac{\mathrm{D}}{\mathrm{D} t}\left(\sum \frac{Y_{\alpha}}{M_{\alpha}} T\right)+(\gamma-1) \frac{\mathrm{D} p}{\mathrm{D} t}\right)^{\prime}
$$

No simplifications are introduced at this stage, i.e., Eq. (23) is valid for the general case of nonisomolar combustion. Here $R_{m}$ denotes the universal gas constant that obeys for ideal gas $R_{m} \sum Y_{\alpha} / M_{\alpha}=R$.

To obtain a formulation of the source term, which can be modeled statistically, fluctuations in the isentropic exponent are neglected, i.e., $\gamma \simeq \bar{\gamma}$. Secondly, only the dominant linear contributions to Eq. (23) are retained. Triple terms expand as

$$
(\rho a b)^{\prime}=\bar{\rho} a^{\prime \prime} \tilde{b}+\bar{\rho} \tilde{a} b^{\prime \prime}+\rho^{\prime} \tilde{a} \tilde{b}
$$

This yields

$$
\begin{aligned}
& q_{p} \simeq \bar{\gamma} \bar{\rho} \tilde{R}\left(\frac{\mathrm{D} T}{\mathrm{D} t}\right)^{\prime \prime}+\bar{\gamma} \bar{\rho} R^{\prime \prime}\left(\widetilde{\mathrm{D} T} \frac{\widetilde{\mathrm{D} t}}{\gamma}+\bar{\gamma} \rho^{\prime} \tilde{R}\left(\widetilde{\mathrm{D} T} \frac{}{\mathrm{D} t}\right)\right. \\
& +(\bar{\gamma}-1)\left(\frac{\mathrm{D} p}{\mathrm{D} t}\right)^{\prime}+\bar{\gamma} R_{m} \bar{\rho} T^{\prime \prime} \sum \frac{1}{M_{\alpha}}\left(\widetilde{\mathrm{D} Y_{\alpha}}\right) \\
& +\bar{\gamma} R_{m} \bar{\rho} \tilde{T} \sum \frac{1}{M_{\alpha}}\left(\frac{\mathrm{D} Y_{\alpha}}{\mathrm{D} t}\right)^{\prime \prime}+\bar{\gamma} R_{m} \rho^{\prime} \tilde{T} \sum \frac{1}{M_{\alpha}}\left(\frac{\widetilde{\mathrm{DY}}}{\mathrm{D} t}\right)
\end{aligned}
$$

The one-point statistics of all quantities that occur in Eq. (25) can be provided by reacting RANS simulations. For statistical noise modeling the two-point cross-correlations of the fluctuating quantities have to be modeled as well. For that, the approximation $\mathrm{D} / \mathrm{D} t \simeq \tilde{\mathrm{D}} / \mathrm{D} t$ with $\tilde{\mathrm{D}} / \mathrm{D} t=\partial / \partial t+\tilde{\boldsymbol{u}} \cdot \nabla$ is applied. Based on this, terms like $(\mathrm{D} \widetilde{T} / \mathrm{D} t)$ and $\left(\mathrm{D} \widetilde{Y_{\alpha} / \mathrm{D} t}\right)$ become

$$
\begin{gathered}
\left(\frac{\widetilde{\mathrm{D} T}}{\mathrm{D} t}\right) \simeq \frac{\tilde{\mathrm{D}} \tilde{T}}{\mathrm{D} t}=\tilde{\boldsymbol{u}} \cdot \nabla \tilde{T} \\
\left(\frac{\widetilde{\mathrm{D} Y_{\alpha}}}{\mathrm{D} t}\right) \simeq \frac{\tilde{\mathrm{D}} \tilde{Y}_{\alpha}}{\mathrm{D} t}=\tilde{\boldsymbol{u}} \cdot \nabla \tilde{Y}_{\alpha}
\end{gathered}
$$

and

$$
\left(\frac{\mathrm{D} T}{\mathrm{D} t}\right)^{\prime \prime} \simeq \frac{\tilde{\mathrm{D}} T^{\prime \prime}}{\mathrm{D} t}
$$

$$
\left(\frac{\mathrm{D} Y_{\alpha}}{\mathrm{D} t}\right)^{\prime \prime} \simeq \frac{\tilde{\mathrm{D}} Y_{\alpha}^{\prime \prime}}{\mathrm{D} t}
$$

Further on

$$
\left(\frac{\mathrm{D} p}{\mathrm{D} t}\right)^{\prime} \simeq \frac{\tilde{\mathrm{D}} p^{\prime}}{\mathrm{D} t}
$$

The final source term of the derived pressure equation, Eq. (18) that comprises reactive flow effects reads

$$
\begin{aligned}
q_{p} & \simeq \bar{\gamma} \bar{\rho} \tilde{R} \frac{\tilde{\mathrm{D}} T^{\prime \prime}}{\mathrm{D} t}+\bar{\gamma} \bar{\rho} R^{\prime \prime} \tilde{\boldsymbol{u}} \cdot \nabla \tilde{T}+\bar{\gamma} \rho^{\prime} \tilde{R} \tilde{\boldsymbol{u}} \cdot \nabla \tilde{T}+(\bar{\gamma}-1) \frac{\tilde{\mathrm{D}} p^{\prime}}{\mathrm{D} t} \\
& +\bar{\gamma} R_{m}\left(\bar{\rho} T^{\prime \prime}+\rho^{\prime} \tilde{T}\right) \sum \frac{1}{M_{\alpha}} \tilde{\boldsymbol{u}} \cdot \nabla \tilde{Y}_{\alpha}+\bar{\gamma} R_{m} \bar{\rho} \tilde{T} \sum \frac{1}{M_{\alpha}} \frac{\tilde{\mathrm{D}} Y_{\alpha}^{\prime \prime}}{\mathrm{D} t}(31)
\end{aligned}
$$

\section{Statistical Source Term Modeling}

For the DLR-A and DLR-B flames considered in this work, which are low Mach number nitrogen-diluted methane-hydrogen flames, it is reasonable to neglect changes in the average molecular weight, i.e., they are well described through isomolar combustion. Hence, the effect of nonisomolar combustion (with $M=\sum Y_{\alpha} / M_{\alpha}=$ const. and $R^{\prime \prime}=0$ ) is neglected and, due to the low Mach number, the sound generated by the turbulent pressure fluctuations. Accordingly, the source term simplifies for this particular case to

$$
q_{p} \simeq \underbrace{\bar{\gamma} \bar{\rho} \tilde{R} \frac{\tilde{\mathrm{D}} T^{\prime \prime}}{\mathrm{D} t}}_{\mathrm{I}}+\underbrace{\bar{\gamma} \tilde{R} \tilde{\boldsymbol{u}} \cdot \nabla \tilde{T} \rho^{\prime}}_{\mathrm{II}}
$$

However, it may be noted that all other contributions to Eq. (31) can be statistically modeled in a similar manner as indicated below for Eq. (32). To show this, in the following the modeling process of both terms of Eq. (32) is discussed. This provides an instructive example as to how the different source terms in Eq. (31) can be realized. However, the first term in Eq. (32) is more prominent than the latter 
because the streamwise variation of mean temperature in term II typically is slow compared with the rapid changes being present in the first term, i.e., if $l_{0}$ is a typical length scale of mean-temperature variation in flow direction, and $\tau_{s}$ is a time scale of the time-gradients of temperature, then $1 / \tau_{s} \gg|\tilde{\boldsymbol{u}}| / l_{0}$. Therefore, the final simulations presented in this paper will be carried out by using only term I in Eq. (32).

Tam and Auriault [20] model the two-point cross correlation of their source term by

$$
\begin{aligned}
& \left\langle\frac{\overline{\mathrm{D}} q_{s}}{\mathrm{D} t}(\boldsymbol{x}, t) \frac{\overline{\mathrm{D}} q_{s}}{\mathrm{D} t}(\boldsymbol{x}+\boldsymbol{\xi}, t+\tau)\right\rangle \\
& =\frac{A}{\tau_{s}^{2}} \exp \left\{-\frac{|\xi|}{u_{c} \tau_{s}}-\frac{\ln (2)}{l_{s}^{* 2}}\left(\left(\xi-u_{c} \tau\right)^{2}+\eta^{2}+\zeta^{2}\right)\right\}
\end{aligned}
$$

where $\xi=(\xi, \eta, \zeta)^{T}$. Constant convection in $x$ direction at velocity $u_{c}$ is considered, and $\tau_{s}$ is a temporal decorrelation time derived from a $k-\epsilon$ model

$$
\tau_{s}=c_{\tau} \frac{k}{\epsilon}
$$

with $c_{\tau}=0.233 . l_{s}^{*}$ is an integral length scale associated to the length scale provided by RANS

$$
l_{s}^{*}=c_{l}^{*} \frac{k^{3 / 2}}{\epsilon}
$$

with $c_{l}^{*}=0.256$. This correlation function, Eq. (33) corresponds to the two-point space-time cross correlation generated by RPM $[\underline{17}, \underline{23}, \underline{25}, 26]$, Eq. (1) if only a constant mean flow in $x$ direction $\overline{u_{c}}=\left(\overline{u_{c}}, \overline{0,0}\right)^{T}$ is used,

$$
\begin{aligned}
& \langle Q(\boldsymbol{x}, t) Q(\boldsymbol{x}+\boldsymbol{\xi}, t+\tau)\rangle=\hat{R}\left\{-\frac{|\tau|}{\tau_{s}}-\frac{\pi}{4 l_{s}^{2}}\left(\left(\xi-u_{c} \tau\right)^{2}\right.\right. \\
& \left.\left.\quad+\eta^{2}+\zeta^{2}\right)\right\}
\end{aligned}
$$

The length scale used here has to be adapted to $l_{s}=$ $l_{s}^{*} \sqrt{\pi / 4 \ln (2)}=1.064 l_{s}^{*}$ to correspond with the original Tam and Auriault length scale. That is, $l_{s}=c_{l} k^{3 / 2} / \epsilon$ with $c_{l}=0.273$. Furthermore, the variance has to be adapted to $\hat{R}=A / \tau_{s}^{2}$. Note, the RPM generated fluctuations satisfy approximately Taylor's convection hypothesis, which allows to substitute $|\xi| / u_{c}$ for $|\tau|$ in Eq. (36) and thus it equals Eq. (33). However, the two-point correlations do not describe strictly frozen turbulence because they include a temporal decorrelation mechanism. Hence, Eqs. (33) and (36) are not exactly equivalent. The small differences are discussed in detail by Morris and Boluriaan [31].

Unlike in the Tam and Auriault model, where only one source term is modeled, the source Eq. (32) comprises two terms, one involving the substantial time derivative of fluctuating temperature, the other one involving simple density fluctuations. Because there is more than one source term, their correlation must be considered when the two-point cross correlation of the complete source $\left\langle q_{p} q_{p}\right\rangle$ is formed. Previous analyses have considered source mechanism to be statistically independent, and that approach, though somewhat questionable, could be followed. Thus, the cross correlation of the source is modeled by

$$
\left\langle q_{p} q_{p}\right\rangle=(\bar{\gamma} \bar{\rho} \tilde{R})^{2}\left\langle\frac{\tilde{\mathrm{D}} T^{\prime \prime}}{\mathrm{D} t} \frac{\tilde{\mathrm{D}} T^{\prime \prime}}{\mathrm{D} t}\right\rangle+(\bar{\gamma} \tilde{R} \tilde{\boldsymbol{u}} \cdot \nabla \tilde{T})^{2}\left\langle\rho^{\prime} \rho^{\prime}\right\rangle
$$

As shown, e.g., by Morris and Farassat [32], the cross correlation of $q_{s}$ itself is

$$
\begin{aligned}
& \left\langle q_{s}(\boldsymbol{x}, t) q_{s}(\boldsymbol{x}+\xi, t+\tau)\right\rangle=A\left\{-\frac{|\xi|}{u_{c} \tau_{s}}-\frac{\ln (2)}{l_{s}^{* 2}}\left(\left(\xi-u_{c} \tau\right)^{2}\right.\right. \\
& \left.\left.\quad+\eta^{2}+\zeta^{2}\right)\right\}
\end{aligned}
$$

if the cross correlation of the substantial time derivative of a quantity $q_{s}$ is described through a model correlation Eq. (33), i.e., it exhibits the same form as Eq. (33) with just the variance adjusted from $A / \tau_{s}^{2}$ to $A$. This is because

$$
\left\langle\frac{\overline{\mathrm{D}} q_{s}}{\mathrm{D} t_{1}}\left(\boldsymbol{x}_{1}, t_{1}\right) \frac{\overline{\mathrm{D}} q_{s}}{\mathrm{D} t}\left(\boldsymbol{x}_{2}, t_{2}\right)\right\rangle=\frac{\overline{\mathrm{D}}^{2}}{\mathrm{D} t_{1} \mathrm{D} t_{2}}\left\langle q_{s}\left(\boldsymbol{x}_{1}, t_{1}\right) q_{s}\left(\boldsymbol{x}_{2}, t_{2}\right)\right\rangle
$$

and the correlation $\mathcal{R}=\left\langle q_{s} q_{s}\right\rangle$ is a function of the form $\mathcal{R}=g(\xi) f\left(\xi-u_{c} \tau\right)$, where $\xi=x_{2}-x_{1}, \tau=t_{2}-t_{1}$, and $\overline{\mathrm{D}} f / \mathrm{D} t=$ 0 if $\overline{\mathrm{D}} / \mathrm{D} t=\partial / \partial t+u_{c} \partial / \partial \xi$, such that $\overline{\mathrm{D}}^{2} \mathcal{R} / \mathrm{D} t_{1} \mathrm{D} t_{2}=u_{c}^{2} f \partial^{2} /$ $\partial \xi^{2} g(\xi)$. With $g(\xi)=\exp \left(-|\xi| / u_{c} \tau_{s}\right)$, it follows $\overline{\mathrm{D}}^{2} \mathcal{R} / \mathrm{D} t_{1} \mathrm{D} t_{2}=$ $1 / \tau_{s}^{2} g f$. For this, it seems reasonable to model the correlations of simple fluctuations and the correlations of quantities that are derived from substantial time differentiation with the same spatiotemporal correlation model.

In the framework of RANS combustion simulations, it is common to solve besides the usual turbulence transport equations, i.e., for $k$ and $\epsilon$, additional transport equations for other variances. For example, transport equations for $\tilde{Y}_{\alpha}^{\prime \prime 2}$ and $\tilde{T}^{\prime \prime 2}$ are solved. Because these equations are formulated in a manner similar to the usual nonreacting flow turbulence equations, where, e.g., dissipation and destruction are controlled by the same turbulent time scale and turbulent viscosity as applied in the $k$ equation, it is deemed reasonable to assume the usual length and time-scale parameters to prescribe the two-point statistics of other variances than $k$ as well. For example, the time scale in the destruction operator of the variance of mass fractions $\tilde{Y}_{\alpha}^{\prime \prime 2}$ is coupled to the turbulent time scale from RANS with a coupling constant of order one [33], whereas in Sec. II.B.3 a coupling constant of 2.0 is used.

In conclusion, to describe the complete source term, Eq. (32) with RPM two mutually uncorrelated generations of fluctuating quantities have to be realized, one for $\tilde{\mathrm{D}} T^{\prime \prime} / \mathrm{D} t$, the other one for $\rho^{\prime}$. Both quantities are modeled with the same formal spatiotemporal correlation function, but their variances are scaled differently. The variances of all quantities are provided by additional RANS transport equations. The time and length scales related to each fluctuating quantity are those deduced from, e.g., the $k-\epsilon$ equations. However, individual calibration parameters $c_{\tau}$ and $c_{l}$ are used for each quantity.

As already mentioned, this discussion intends to show how both source terms in Eq. (32) can be generated stochastically. This example can be used as a paradigm to model the remaining source terms in Eq. (31) as well1. However, for the work presented in this paper no additional transport equation for the fluctuating density variance was implemented and its contribution to the combustion sound source will be neglected further on. An extra transport equation is solved for the temperature variance $\tilde{T}^{\prime \prime 2}$ and this quantity is directly associated with $A$ in Eq. (33), i.e., one RPM variable is realized with variance $\hat{R}=\tilde{T}^{\prime \prime 2} / \tau_{s}^{2}$.

\section{System of Equations}

The pressure equation derived above is equal to the LHS of the linearized Euler pressure equation and considered in combination with the continuity and the momentum equation of the genuine linearized Euler equations (LEE). Because reacting RANS simulations provide Favre-averaged velocities, these are used instead of time-averaged velocities in the LEE. The effect of Favre- versus Reynolds-averaged velocities on wave propagation physics is deemed to be small, because the jet velocities in the flame are below $M a=0.2$ and the main refraction effect is caused by the large density gradients, which are properly used as Reynolds-averaged quantities. Hence, the acoustic model applied in this work is given by

\footnotetext{
IOne peculiar detail that would come into picture for the remaining terms of Eq. (32) would be that the $\tilde{Y}_{\alpha}^{\prime \prime}$ correlations are mutually interconnected via the chemical reaction equations. This mutual correlation can be considered in the RPM modeling, however, a detailed discussion is beyond the scope of this paper.
} 


$$
\begin{array}{r}
\frac{\partial \rho^{\prime}}{\partial t}+\tilde{\boldsymbol{u}} \cdot \nabla \rho^{\prime}+\boldsymbol{u}^{\prime} \cdot \nabla \bar{\rho}+\bar{\rho} \nabla \cdot \boldsymbol{u}^{\prime}+\rho^{\prime} \nabla \cdot \tilde{\boldsymbol{u}}=0 \\
\frac{\partial \boldsymbol{u}^{\prime}}{\partial t}+(\tilde{\boldsymbol{u}} \cdot \nabla) \boldsymbol{u}^{\prime}+\left(\boldsymbol{u}^{\prime} \cdot \nabla\right) \tilde{\boldsymbol{u}}+\frac{\nabla p^{\prime}}{\bar{\rho}}-\frac{\nabla \bar{p} \rho^{\prime}}{\bar{\rho}^{2}}=\mathbf{0} \\
\frac{\partial p^{\prime}}{\partial t}+\tilde{\boldsymbol{u}} \cdot \nabla p^{\prime}+\boldsymbol{u}^{\prime} \cdot \nabla \bar{p}+\gamma \bar{p} \nabla \cdot \boldsymbol{u}^{\prime}+\gamma p^{\prime} \nabla \cdot \tilde{\boldsymbol{u}}=q_{p}
\end{array}
$$

with the combustion noise source term

$$
q_{p}=\frac{\bar{\gamma} \bar{p}}{\tilde{T}} \frac{\tilde{\mathrm{D}} T^{\prime \prime}}{\mathrm{D} t}
$$

The two-point cross correlations of the substantial time derivative of the temperature fluctuations in Eq. (43) are modeled with Eq. (33). Hence, as an ansatz for the variance of the substantial time derivative of the temperature fluctuations, a term proportional to the variance of the Favre-averaged temperature fluctuations divided by a turbulent combustion time scale $\tau_{T}$ squared is used. Furthermore an additional calibration constant $c_{q}$ is considered, that is

$$
\hat{R}=\frac{\tilde{T}^{\prime \prime 2}}{c_{q}^{2} \tau_{T}^{2}}
$$

The use of Eq. (44) in conjunction with Eq. ()ㅡㄹ yields the amplitude $\hat{A}$, where $n$ indicates the dimension of the problem

$$
\hat{A}=\frac{1}{c_{q} \tau_{T}} \sqrt{\frac{\rho_{0}^{c} \tilde{T}^{\prime \prime 2}}{l_{T}^{n}}}
$$

The modeled correlation function has three characteristic parameters, which are the length scale $l_{T}$ and time scale $\tau_{T}$ of the turbulent temperature fluctuations as well as the temperature variance $\tilde{T}^{\prime \prime 2}$. Note that the turbulent temperature length $l_{T}$ and time scales $\tau_{T}$ might differ from the length $l_{s}$ and time scales $\tau_{s}$ used, e.g., for the Tam and Auriault jet noise model [20]. This means that different calibration constants have to be applied. To clearly distinguish the turbulent temperature scales, the related parameters are labeled $l_{T}$, $\tau_{T}, c_{T l}$, and $c_{T \tau}$, instead of $l_{s}, \tau_{s}, c_{l}$, and $c_{\tau}$ in the following. This convention already has been used in Eqs. (44) and (45), where the length and time scale $l_{T}$ and $\tau_{T}$ instead of $l_{s}$ and $\tau_{s}$ occur. The turbulent temperature length $l_{T}$ and the time scale $\tau_{T}$ are linked to the turbulence model scales by

$$
\begin{gathered}
l_{T}=c_{T l} \frac{k^{3 / 2}}{\epsilon} \\
\tau_{T}=c_{T \tau} \frac{k}{\epsilon}
\end{gathered}
$$

For the computation of the temperature variance a transport equation [33] is solved

$$
\begin{aligned}
& \frac{\partial \bar{\rho} \tilde{T}^{\prime \prime 2}}{\partial t}+\frac{\partial \bar{\rho} \tilde{u}_{j} \tilde{T}^{\prime \prime 2}}{\partial x_{j}}=\frac{\partial}{\partial x_{j}}\left\{\left(\bar{\mu}+\frac{\mu_{t}}{P r_{t}}\right) \frac{\partial \rho \tilde{T}^{\prime \prime 2}}{\partial x_{j}}\right\}+c_{\text {prod }} \frac{\mu_{t}}{P r_{t}}\left(\frac{\partial \tilde{\bar{T}}}{\partial x_{j}}\right)^{2} \\
& -c_{\text {diss }} \overline{\bar{\rho}} \frac{\bar{\epsilon}}{\tilde{k}} \tilde{T}^{\prime \prime 2}
\end{aligned}
$$

with $c_{\text {prod }}=2.0$ and $c_{\text {diss }}=2.0$.

To finally obtain the source term, Eq. (43) the RPM generated source has to be complemented with the prefactor $\bar{\gamma} \bar{p} / \tilde{T}$, which is deduces from the reacting RANS simulation.

\section{Discussion}

Next, the suitability of the RPM method for modeling combustion noise sources will be discussed. The RPM generated correlations of the scalar source, Eq. (43) will be Gaussian. Currently, no data is available concerning the realistic shape of the correlation function associated to the derived combustion noise source term. The acoustic effect of the length scale is to distinguish between coherent and incoherent source fluctuations. If the turbulent length scale is smaller than the acoustic wavelength, coherent fluctuations can be deemed to be acoustically compact. This effectively means that only the local correlation length scale is acoustically important, but not the specific shape of the correlation function. The assumption of compactness is satisfied with good accuracy for combustion noise problems at low Mach numbers. Hence, a Gaussian spatial correlation of the combustion source might be an appropriate ansatz for its shape. Another issue is the probability density function (PDF) of the turbulent temperature fluctuations. RPM generates fluctuations with Gaussian PDF. This aspect corresponds very well with experimental observations. Gerlinger et al. [34] analyzed one-dimensional marginal PDFs of temperature of a subsonic hydrogen jet flame [21], for example. In total, 300 single shot data were evaluated, and the PDF structures calculated. Most temperature PDFs exhibited approximately Gaussian distribution.

Furthermore, the characteristics of the derived combustion noise source term are discussed. Ihme et al. [2] report about the dominant acoustic effect of the heat release rate using LES as a basis to describe sound sources for CAA. Hirsch et al. [8] successfully use a model for the spectral distribution of the heat release in turbulent premixed combustion to calculate sound spectra. However, Bui et al. [35] use the scaled substantial and partial time derivatives of density as alternative combustion source terms for CAA, which were shown to encompass the heat release rate together with other source contributions. By using LES data to describe the sources, they compare the spectra based on their more complete combustion sound sources with that of the heat release term only and find a drop in level of almost $6 \mathrm{~dB}$ over the entire spectral range for the latter. It is probable that the heat release term alone is not sufficient for an accurate simulation of absolute sound pressure levels. The derived combustion noise source term of the RPM-CN approach includes further thermoacoustic sources besides the fluctuating heat release. Furthermore, the modeling of the combustion noise source as a function of the temperature fluctuations provides the possibility to validate the temperature statistics by experimental data.

Finally, the derived LEE based RPM combustion noise model includes some beneficial features compared with the APE based model used in a previous paper [18]. The LEE support an entropy mode, which allows for a direct simulation of indirect combustion noise due to convective turbulence related hot spots. Entropy fluctuations are excited automatically by the direct combustion noise source term, Eq. (43). Furthermore, the homogeneous LEE are suited to exactly resolve the shear layer refraction effects that occur in combustion problems, i.e., in mean flows with strong velocity and/or density gradients. Because the LEE realize an exact solution of the conservation equations for small disturbances, the LEE refraction result can be deemed to be close to the exact physical solution.

\section{Experimental Test Case and Numerical Setup}

\section{A. DLR-A and DLR-B Flame}

Two benchmark flames (DLR-A and DLR-B) of the International Workshop on Measurement and Computation of Turbulent Nonpremixed Flames [21] are used to validate the proposed RPM$\mathrm{CN}$ approach. In both cases the configuration was a open, nonpremixed, turbulent nitrogen-diluted methane-hydrogen jet flame. The fuel with a composition of $22.1 \% \mathrm{CH}_{4}, 33.2 \% \mathrm{H}_{2}$ and $44.7 \% \mathrm{~N}_{2}$, and a stoichiometric mixture fraction of 0.167 was injected through a $0.35 \mathrm{~m}$ long straight stainless steel tube of diameter $D=0.008 \mathrm{~m}$. The mean jet exit velocity corresponded to $42.15 \mathrm{~m} / \mathrm{s}$ for the DLR-A flame, to $63.2 \mathrm{~m} / \mathrm{s}$ for the DLR-B flame and the temperature in both cases to $292 \mathrm{~K}$. The Reynolds number with respect to $D$, the pipe bulk exit velocity and kinematic viscosity of the fuel mixture was $R e=$ 15200 and $R e=22800$, respectively. The tube was surrounded by a contoured nozzle supplying coflowing dry air at an exit velocity of $0.3 \mathrm{~m} / \mathrm{s}$ at a temperature of $292 \mathrm{~K}$. The outer nozzle had a diameter 
of $0.14 \mathrm{~m}$. The configurations have been experimentally investigated by Bergmann et al. [36], Meier et al. [37] and Schneider et al. [38]. The spectral sound emissions were measured by Singh et al. [39].

\section{B. CFD Setup}

The CFD simulations were performed applying the commercial software package ANSYS CFX 11. The fully three dimensional computational domain was discretized with a cylindrical unstructured hexahedron grid consisting of 370000 grid nodes. The grid had dimensions of 94D in axial and 113D in radial direction and was strongly refined in the combustion zone to resolve the strong gradients of the flow field. Furthermore, the growth of the adjacent cells was limited to $10 \%$. The fuel supply was modeled by a velocity inlet boundary condition. The measured velocity profile and profiles of the turbulence kinetic energy and the turbulence dissipation rate of the DLR-A and the DLR-B flames were specified at a temperature of $295 \mathrm{~K}$. The coflow was defined by a mass flow of $5.5 \mathrm{~g} / \mathrm{s}$ at a temperature of $295 \mathrm{~K}$. The walls of the fuel pipe and the coflow were modeled by a adiabatic no-slip wall boundary condition. The freestream boundaries of the computational domain were approximated by opening boundary conditions applying a static pressure of $0 \mathrm{~Pa}$.

A fully three dimensional compressible steady-state RANS simulation was performed to calculate the reacting flow field. The turbulence was considered by the baseline Reynolds stress model (BSL-RSM) [40]. Chemical reactions were simulated applying the burning velocity model [41], whereas the turbulent flame velocity was computed using the Zimont correlation [42]. Additionally, a transport equation for the temperature variance, $\overline{\mathrm{Eq}}$. (48) was solved. The fully implicit solver is based on a finite volume formulation for unstructured grids. The linear set of coupled equations was solved by a multigrid strategy. For the spacial discretization the high resolution scheme was used, which is a bounded second-order upwind based discretization. Furthermore, buoyancy was taken into account, whereas heat radiation was neglected.

\section{CAA Setup}

The acoustic processes were simulated by solving the LEE equations, which were integrated by the DLR-CAA code PIANO [43]. The stochastic reconstruction of the broadband combustion noise sources is implemented in a development version of the PIANO code. Currently, the implementation of the stochastic source term reconstruction is restricted to two dimensional modeling. Therefore, the computational CAA domain was discretized by a two dimensional plane block structured grid of 97000 grid nodes, which was refined in the acoustic source zone. The growth of the adjacent cells was limited to $10 \%$. The mesh covered $90 \mathrm{D}$ in axial and $110 \mathrm{D}$ in radial direction and was appropriate to solve acoustic frequencies up to $f=11000 \mathrm{~Hz}$ based on seven points per wavelength. Acoustic nonreflective radiation boundary conditions by Tam and Webb [44] surrounded the computational domain. The PIANO code applies the fourth-order DRP scheme of Tam and Webb [44] in space and a LDDRK method [45] in time on block structured meshes. The chosen time step size of $2 \cdot 10^{-6}$ s allowed a resolution of frequencies up to $f=71000 \mathrm{~Hz}$ based on 7 points per period (PPP).

\section{Results}

\section{A. Reacting Flow}

The computed axial profiles on the center line of the axial velocity, mixture fraction, temperature, and temperature RMS of the DLR-A flame are compared with experimental data in Fig. 1 . Figure 2 shows the simulated profiles of the axial velocity, mixture fraction, temperature, and temperature RMS of the DLR-B flame in comparison with measurements. The measured axial velocity profile of the DLR-B flame is not available. The reference diameter is the nozzle diameter $D=0.008 \mathrm{~m}$ and the mixture fraction was calculated using Bilger's definition [46].

The computed axial velocity profile of the DLR-A flame exhibits a good agreement with the experimental data for $x / D<20$ and $x / D>60$. In the axial range $20<x / D<60$ the computed profile shows certain deviations compared with the measurements. The axial profile of the mixture fraction is in good agreement with the the experimental data. However, discrepancies are also found in the axial range of $20<x / D<60$. Consequently, the simulated temperature profile agrees reasonably well with measurements. Along the center line the simulated maximal temperature is slightly overpredicted by $46 \mathrm{~K}$. The transport equation of the temperature variance in combination with the RANS simulation delivers satisfactory results. The maximum value of the temperature RMS agrees well with the experimental data. The predicted profiles of the DLR-B flame show qualitatively similar agreement with the experimental data as the discussed computational results of the DLR-A flame. In this case, the simulated maximal temperature is overpredicted by $41 \mathrm{~K}$ along the center line.

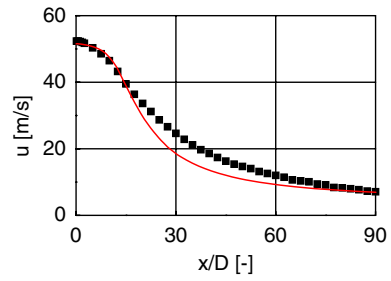

a) Axial velocity

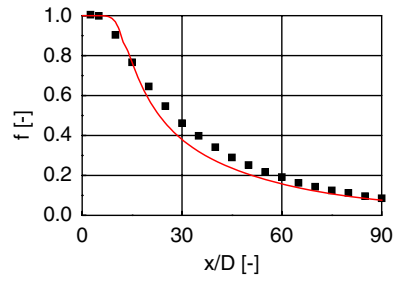

b) Mixture fraction

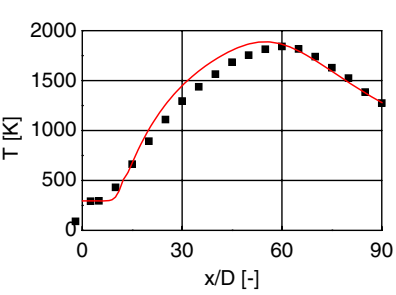

c) Temperature

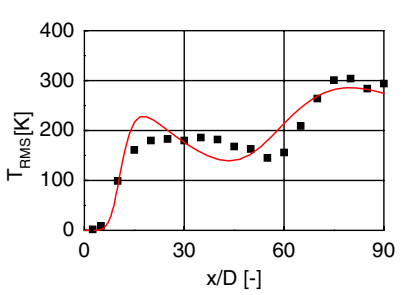

d) Temperature RMS

Fig. 1 Axial profiles at $y / D=0$ of the axial velocity, mixture fraction, temperature, and temperature RMS of the DLR-A flame. The dotted line denotes experiment, and the solid line denotes simulation.

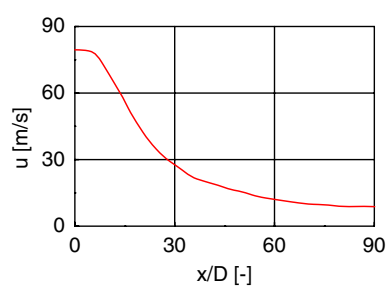

a) Axial velocity

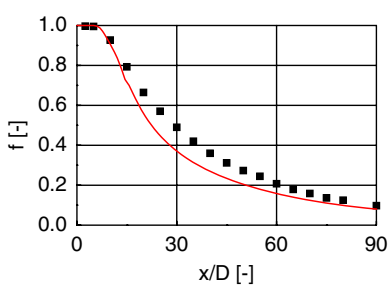

b) Mixture fraction

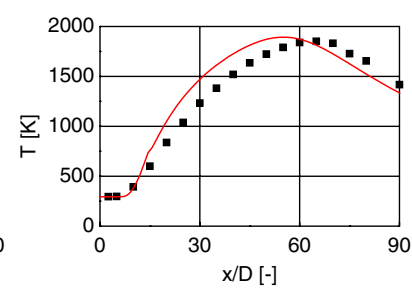

c) Temperature

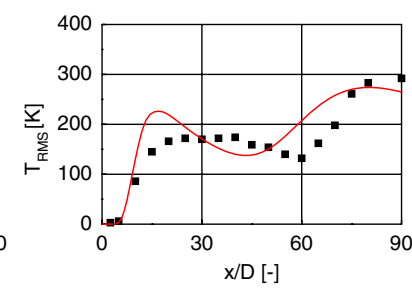

d) Temperature RMS

Fig. 2 Axial profiles at $y / D=0$ of the axial velocity, mixture fraction, temperature, and temperature RMS of the DLR-B flame. The dotted line denotes experiment, and the solid line denotes simulation. 
Detailed investigations showed that the deviations of the simulated velocity field are not dependent on the boundary conditions or on the quality of the combustion model. Simulations using a refined grid existing of 2,100,000 grid nodes did not show a relevant improvement concerning the agreement with measurements compared with the base grid. Different turbulence models have been tested, and it has been found that the BSL-RSM model provided the best results in this case. It is sometimes observed that standard RANS turbulence models perform poorly in predicting the mean flow of jets. However, modified two-equation RANS models have been developed recently that show good prediction quality for jet flows [47]. However, the applied RANS BLS-RSM model approach delivers acceptable results at low computational costs. Deviations of the mixture fraction can be attributed to the suboptimal simulation of the velocity field. The remaining slight overprediction of the maximal temperature can be due to turbulent mixing effects or the neglect of heat radiation. Deviations of the temperature RMS, compared with experimental data can additionally be caused by turbulence modeling and combustion modeling because the temperature variance transport equation, Eq. (48) is a function of the turbulence kinetic energy, the turbulence eddy dissipation and the temperature.

\section{B. Acoustics}

In a first step, an evaluation was performed regarding the capability of the RPM method to realize the defined two-point correlations and to reconstruct the resolution of the target source variance. Figure 3 shows the statistical analysis of fluctuating combustion noise sources from RPM for a constant convection velocity in $x$ direction $u_{c}=0.04$, constant length scale $l_{T}=0.0005$,

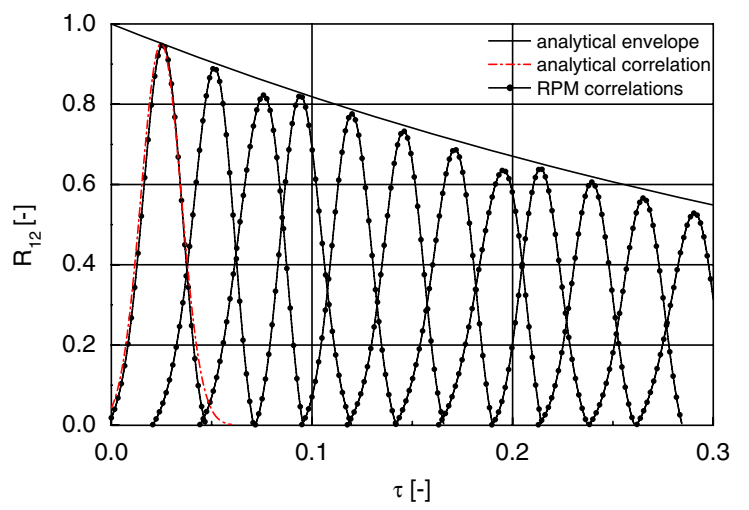

Fig. 3 Two-point space-time correlations of the RPM sources, analytical correlation and analytical envelope. and constant time scale $\tau_{T}=0.5 . \mathcal{R}_{12}$ denotes the cross correlation between two sampling points inside the generic test field. The cross correlation between two sampling points $i$ for a relative time shift $\tau$ is evaluated from the unsteady time samples $Q_{i}\left(x_{i} t\right)$ by integrating over the sampling interval $\Delta T$, that is

$$
\begin{aligned}
& \mathcal{R}_{12}(\tau)=\overline{Q_{1}\left(x_{1}, t\right) Q_{2}\left(x_{2}, t+\tau\right)}=\frac{1}{\Delta T} \int_{T_{0}}^{T_{0}+\Delta T} \\
& \quad \times Q_{1}\left(x_{1}, t\right) Q_{2}\left(x_{2}, t+\tau\right) \mathrm{d} t
\end{aligned}
$$

All $i=12$ points used are distributed with equal spacing $\Delta x=0.001$ along the $x$-axis. Starting from the farthest left cross-correlation curve, the sequence of correlation curves refer to the crosscorrelations between point combinations 1-2 up to 1-12.

Figure 3 compares RPM generated correlations with the spatial target correlation and the target time decorrelation. The comparison shows that two-point correlations can be reconstructed very well with the RPM method. In other words, for a given combination of two sampling points, the related cross-correlation curves have Gaussian shape. The realized two-point correlation of the RPM method shows a good agreement with the analytical correlation according to Eq. (1). Furthermore, the maximum of the achieved correlation decays with the second sampling point farther downstream chosen. The envelope of all curves has exponential behavior and only small deviations compared with the analytical envelope $\exp \left(-|\tau| / \tau_{T}\right)$ can be determined. The horizontal location of the cross-correlation maximum is successively shifted to higher time shifts $\tau$ for the cross correlation with the second sampling point chosen farther downstream. The constant time difference of $\Delta \tau=0.025$ between the maxima indicates the achievement of a constant convection velocity for the equidistantly distributed sampling points. Based on the constant time difference $\Delta \tau=0.025$ as indicated in Fig. 3 and the equidistant spacing $\Delta x=0.001$ between the sampling points, the defined constant convection velocity in $x$ direction $u_{c}=0.04$ can be exactly determined.

Another important feature that has to be accomplished by the stochastic model is the accurate resolution of the source variance $\hat{R}$, Eq. (44) topology. Figure 4 juxtaposes the RANS target solution of the source variance to the corresponding results from the stochastic modeling and an instantaneous RPM source distribution. For this purpose, 100,000 time levels of the stochastically generated sources are sampled and averaged. A good realization of the source variance for the energy topology as well as the absolute magnitudes is found. The small deviations of the modeled source variance distribution from the target source variance can be attributed to insufficient averaging.

In Figs. 5- $\underline{8}$ the computed sound pressure level spectra of the DLR-A and the DLR-B flame at $x / D=0$ and $y / D=25$ are

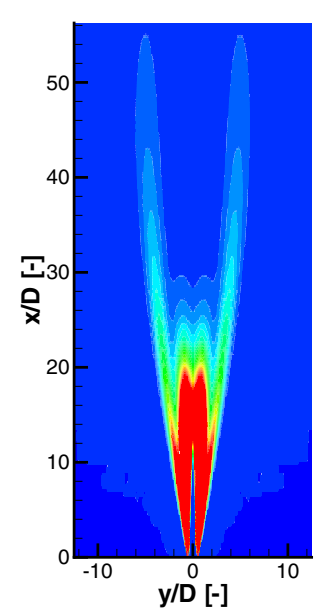

a) RANS

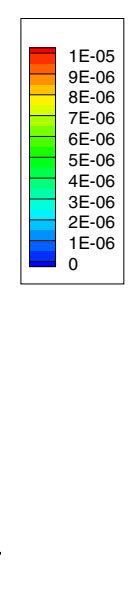

b) RPM

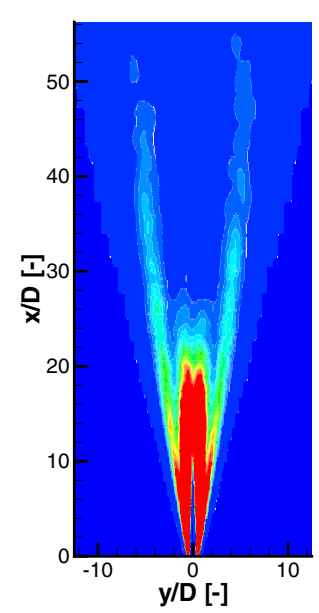

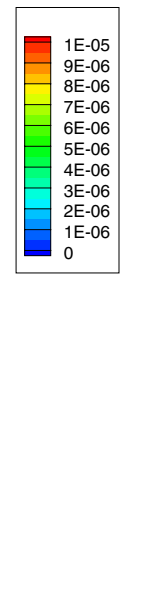

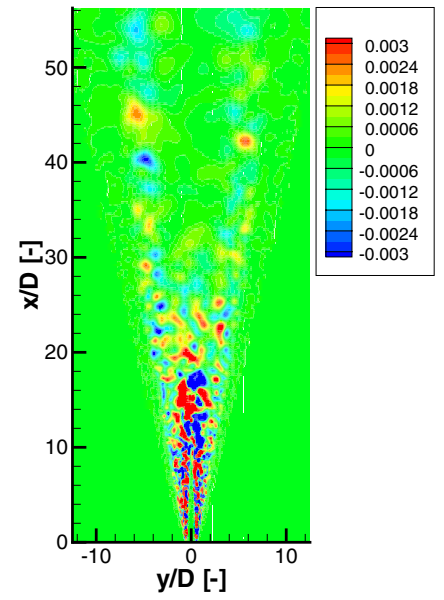

c) RPM sources

Fig. 4 RANS target source variance distribution, source variance distribution of the stochastic reconstruction by RPM, and an instantaneous RPM source distribution. 


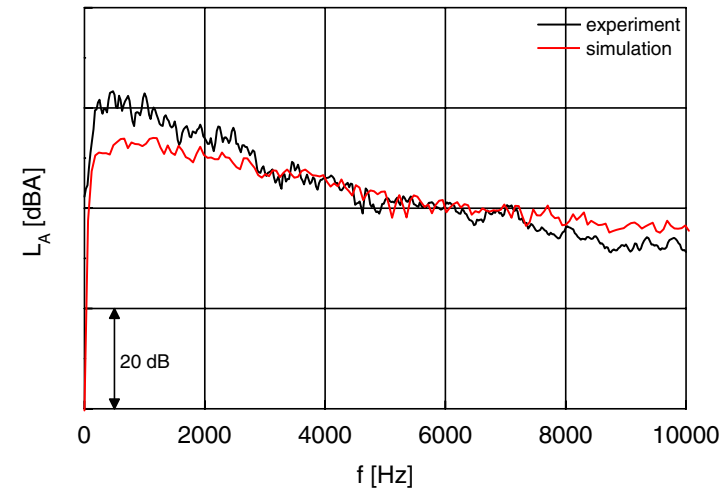

a) DLR-A

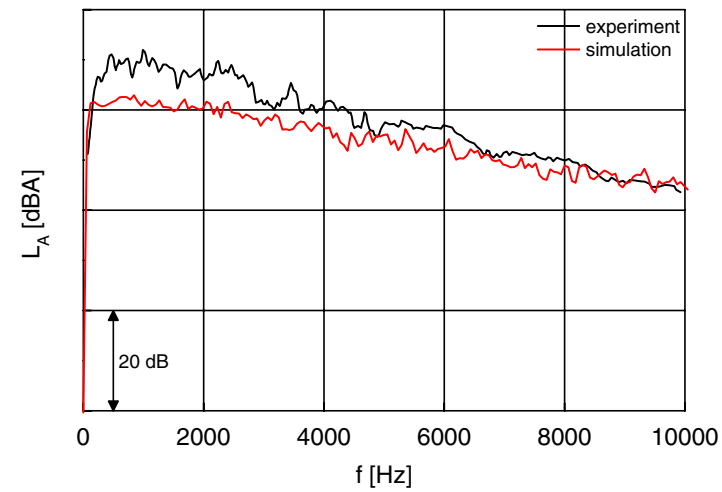

b) DLR-B

Fig. 5 Computed and measured sound pressure level spectra of the DLR-A and the DLR-B flame. The constants $c_{T l}=0.273$ and $c_{T \tau}=0.233$ were defined in accordance to the Tam and Auriault [20] RPM realization for jet noise simulation $[\underline{17}, \underline{23}, \underline{25}]$.

compared with the experimental data of Singh et al. [39]. Narrowband spectra were obtained by a fast Fourier transformation (FFT) of the computed pressure signals. A real time of $0.02 \mathrm{~s}$ was evaluated and 10 averages were used. According to the NyquistShannon sampling theorem, the resolution of frequencies below $f<100 \mathrm{~Hz}$ is excluded due to the length of the evaluated time signal. A frequency resolution of $25 \mathrm{~Hz}$ was chosen for the FFT and the sound pressure level spectra were A-weighted decibel (dBA), both in accordance to the experimental data [39]. The A-weighting filter rolls off below $1 \mathrm{kHz}$, by about $4 \mathrm{~dB}$ at $400 \mathrm{~Hz}$ and features a slight gain above $1 \mathrm{kHz}$, about $4 \mathrm{~dB}$ at $5 \mathrm{kHz}$.

Preliminary investigations proved that the numerical setup is independent on the source domain size, the number of streamlines spanning the source domain, the number of random particles drifting along the streamlines, the seeding clock rate of the particles, and the time step size of the acoustic computation.

Because of the absence of available data concerning the typical shape of the correlation function associated with the used combustion noise source term, Eq. (43) the parameters $c_{T l}$ and $c_{T \tau}$, Eqs. (46) and (47) of the RPM-CN approach have to be calibrated. Note, the modeling constants $c_{T l}$ and $c_{T \tau}$ do not have to be specified in LES/DNS like approaches, were turbulence is time accurately resolved $[2,35,48]$. As a starting point the parameters were defined in accordance with the Tam and Auriault [20] RPM realization for jet noise simulation $[17,23,25]$. Hence, the computed spectra of the DLR-A and the DLR-B flame displayed in Fig. 5 have been obtained by applying the parameters $c_{T l}=0.273$ and $c_{T \tau}=0.233$. Because the amplitude is not scaled yet in the source term modeling for the

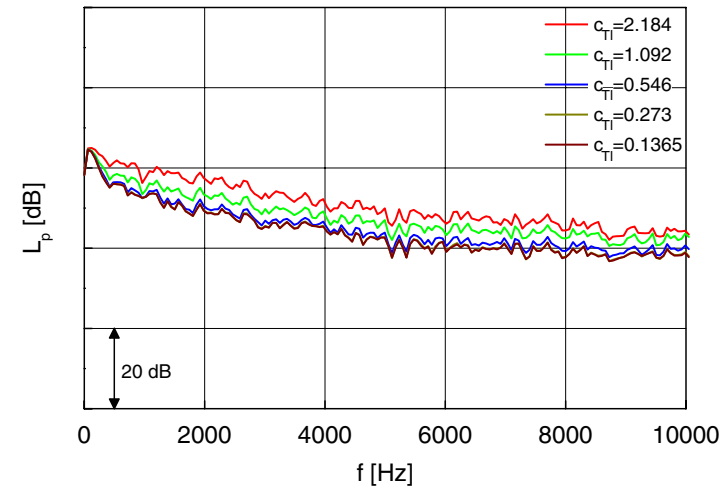

a) DLR-A

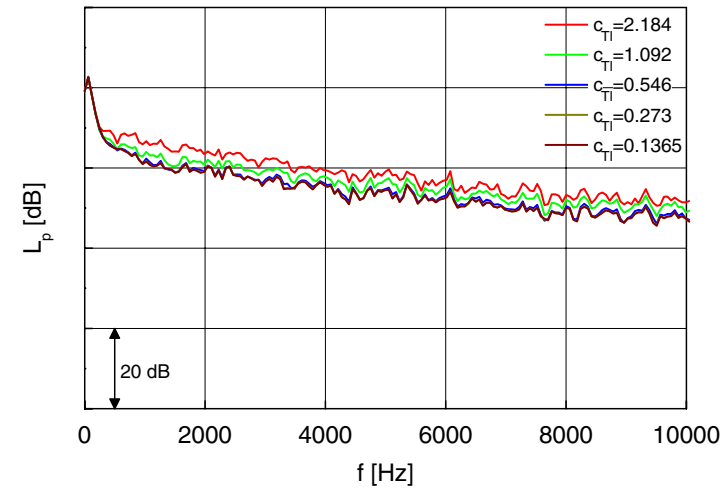

b) DLR-B

Fig. 6 Computed sound pressure level spectra of the DLR-A and the DLR-B flame for different $c_{T l}$.

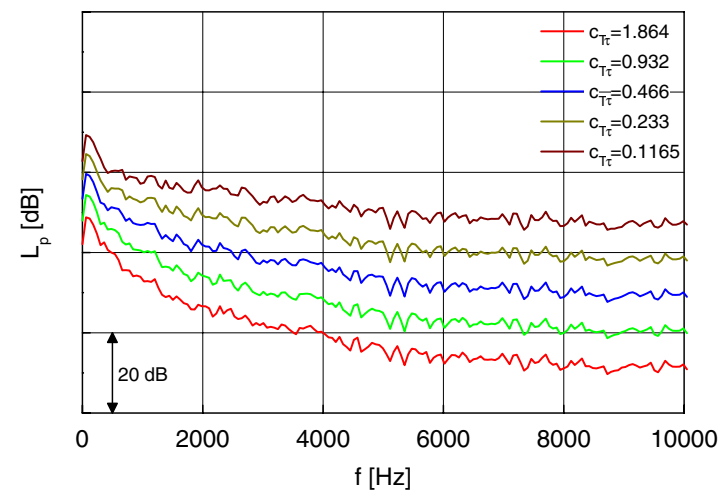

a) DLR-A

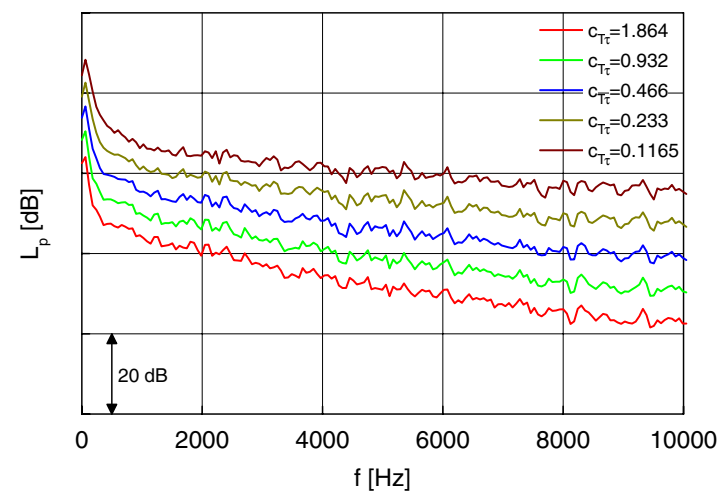

b) DLR-B

Fig. 7 Computed sound pressure level spectra of the DLR-A and the DLR-B flame for different $c_{T \tau}$. 

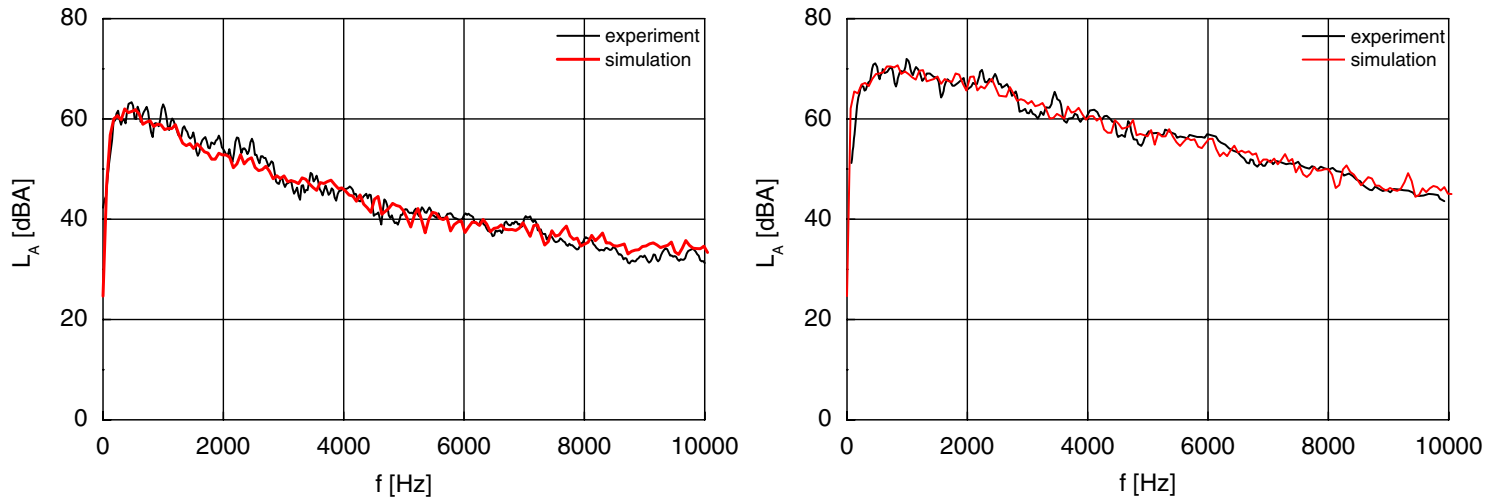

a) DLR-A

b) DLR-B

Fig. 8 Computed and measured sound pressure level spectra of the DLR-A and the DLR-B flame. The optimized parameters $c_{T l}=0.273$ and $c_{T \tau}=1.864$ were applied.

case of two dimensional computation, the sound pressure level spectra are not comparable to measurements in terms of absolute levels. The shapes of both predicted sound pressure level spectra show larger deviations in comparison with the experimental data. This is not a surprise, because it should not be expected that the integral length and time scale of a combustion sound source are exactly equivalent to that of a cold jet noise sound source. Hence, the parameters of the RPM-CN approach have to be calibrated.

Figures 6 and 7 depict numerical sensitivity analysis of the parameters $c_{T l}$ and $c_{T \tau}$ to indicate the influence on the computed sound pressure level spectra of the DLR-A and the DLR-B flame. The sound pressure level spectra are depicted without A-weighting to exclude the influence of the filter function on the parameter study. The computed sound pressure level spectra in Fig. 6 indicate for the DLR-A and more pronounced for the DLR-B flame a slight change of the shape in the low-frequency range because of the absence of the A-weighting. Above approximately $1000 \mathrm{~Hz}$ the shape remains nearly unchanged and there is a shift of the amplitude observable. The variation of $c_{T \tau}$ affects the shape as well as the amplitude of the computed sound pressure level spectra. With increasing $c_{T \tau}$ the amplitudes decreases, and the shape of the spectra indicate a stronger decline towards higher frequencies. The amplitude function $\hat{A}$, Eq. (45) is a function of the reciprocal value of the time scale $\tau_{T}$ and the parameter $c_{T \tau}$, respectively. Additionally, the temporal decay $\exp \left(-|\tau| / \tau_{T}\right)$ according to Eq. (1) is affected by the parameter $c_{T \tau}$ scaling the time scale $\tau_{T}$. Increasing $c_{T \tau}$ results to a slower temporal exponential decay and subsequently a stronger decline of the sound pressure level spectra towards higher frequencies.

To determine the optimal parameter set for the combustion noise simulation of the DLR-A and DLR-B flame, a least-square approach was employed. Therefore, a set of preliminary simulations was performed. Each simulation was conducted with a different

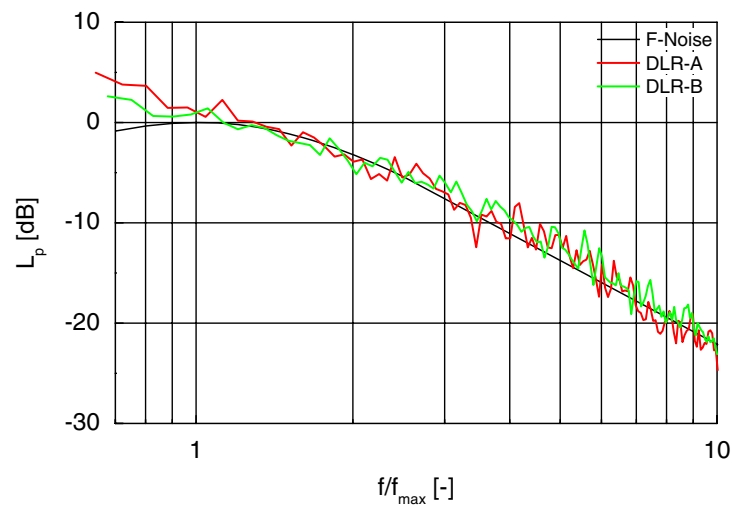

Fig. 9 F-Noise similarity spectrum of Tam et al. [49] and the computed sound pressure level spectra of the DLR-A and the DLR-B. The optimized parameters $c_{T l}=0.273$ and $c_{T \tau}=1.864$ were applied. parameter setting, yielding a corresponding course of the sound pressure level spectrum. To obtain the best fit between simulation and experiment a linear correlation between the parameters and the resulting sound pressure level spectra is assumed. This leads to a least-square formulation, which can be solved for any of the parameters. Hence, the results displayed in Fig. 8 have been obtained applying the optimized parameters $c_{T l}=0.2 \overline{73}$ and $c_{T \tau}=1.864$. The computed sound pressure level spectra of the DLR-A and the DLR-B flame are both in excellent agreement with the measurements over the entire frequency range, Fig. $\underline{8}$. The shape as well as the amplitude of the predicted spectra show deviations compared with the experimental data of the order of the natural fluctuations present in the narrow band spectra.

Tam et al. [49] presented extensive experimental measurements to demonstrate that combustion noise from auxiliary power units (APU) has a unique shape. The spectral shape which was found is the same as the F-Noise similarity spectrum of large turbulence structures noise of high-speed jets [50]. Furthermore, Tam et al. [49] observed that the identified unique spectral shape of combustion noise of APUs is virtually the same as that of open flame combustion noise. In Fig. 9 the computed combustion noise spectra of the DLR-A and DLR-B flame are compared with the suggested similarity spectrum. For both flames, the simulated spectrum indicates a decay for higher frequencies, which is consistent with the F-Noise similarity spectrum. The decay in the low-frequency range of the RPM-CN spectra deviates from the F-Noise spectrum. These deviations probably can be attributed to a too short sampling time.

The CFD simulations have been performed in parallel processing on two IBM Power5 processors. Thus, the cost of a simulation was approximately $11 \mathrm{cpu}$ hours. The subsequent combustion noise source modeling and the acoustic simulations were performed sequentially on a $2.0 \mathrm{GHz}$ processor requiring $35 \mathrm{cpu}$ hours on average.

\section{Conclusions}

The derived and validated RPM-CN approach is a highly efficient hybrid CFD/CAA method for the numerical simulation of broadband combustion noise. The approach relies on the stochastic reconstruction of combustion noise sources in the time domain out of statistical turbulence quantities which can be delivered by reacting RANS simulations. The modeled combustion noise sources are derived for the use in conjunction with the LEE for the computation of the acoustic propagation.

Two open, nonpremixed, turbulent jet flames (DLR-A and DLRB) were used for the validation of the RPM-CN approach. The two flames are similar except for their different fuel outlet velocity and their respective Reynolds number. First, the results of the reacting RANS computations of both flames were presented and discussed. Subsequently, the capability of the RPM method to reproduce the prescribed two-point correlations was demonstrated. A good realization of the target source variance by the RPM method was 
achieved. The influence of the length and time scaling parameters of the turbulence temperature fluctuations on the predicted sound pressure level spectra was demonstrated by sensitivity analyses. Finally, the simulated spectra for the optimized model parameters $c_{T \tau}$ and $c_{T l}$ of the DLR-A and the DLR-B flame were compared with measurements. The spectra show an excellent agreement with the experimental data. Furthermore, it was shown, that the combustion noise spectra of the DLR flames predicted by the RPM-CN approach follow the F-Noise similarity spectrum proposed by Tam et al. [49].

Although, the stochastic source reconstruction and therefore the acoustic computation has been restricted to a two dimensional implementation until now, the good agreement of numerical simulations and experimental data indicates the reliability and the Reynolds scalability of the numerical approach. In a next step we will study the application of the simulation method to confined combustion chambers. A fast noise prediction technique that can be applied to study the acoustic features of confined flames would be of great value for manufacturers to enable the consideration of aeroacoustic questions during the design phase of combustion chambers. For design applications, however, it has to be proven that the model parameters $c_{T l}$ and $c_{T \tau}$ remain nearly constant at least for a certain class of problems, when geometrical and physical parameters are varied. Otherwise, it would not be possible to predict deltas of sound pressure levels between different designs properly. In this regard, the successful prediction of two unconfined flames in this work using a common set of parameters is an important step forward. In combination with very low computational costs the application of the highly efficient RPM-CN approach could be useful for aeroacoustics driven design.

\section{Acknowledgments}

This collaborative work was supported within the project AVANTGARDE, which is funded by DLR, German Aerospace Center. The authors would like to thank Christian Eberle and Jan Boyde from the DLR Institute of Combustion Technology in Stuttgart, Germany for their contribution to this work.

\section{References}

[1] Bui, T., Schröder, W., and Meinke, M., "Numerical Analysis of the Acoustic Field of Reacting Flows via Acoustic Perturbation Equations," Computers and Fluids Journal, Vol. 37, No. 9, 2008, pp. 1157-1169. doi:10.1016/j.compfluid.2007.10.014

[2] Ihme, M., Bodony, D., and Pitsch, H., "Prediction of CombustionGenerated Noise in Non-Premixed Turbulent Jet Flames Using Large-Eddy Simulation," AIAA Paper 2006-2614, Cambridge, MA, 2006.

[3] Flemming, F., Sadiki, A., and Janicka, J., "Investigation of Combustion Noise Using a LES/CAA Hybrid Approach," Proceedings of the Combustion Institute, Vol. 31, Elsevier, Amsterdam, 2007, pp. 3189 3196.

[4] Brick, H., Piscoya, R., Ochmann, M., and Költzsch, P., "Prediction of the Sound Radiation from Open Flames by Coupling a Large Eddy Simulation and a Kirchhoff Method," Acustica, Vol. 91, 2005, pp. 1721.

[5] Lyrintzis, A., "Integral Acoustics Methods: From the (CFD) Near- Field to the (Acoustic) Far-Field," International Journal of Aeroacoustics, Vol. 2, No. 2, 2003, pp. 95-128. doi: $10.1260 / 147547203322775498$

[6] Béchara, W., Bailly, C., Lafon, P., and Candel, S., "Stochastic Approach to Noise Modelling for Free Turbulent Flows," AIAA Journal, Vol. 32, No. 3, 1994, pp. 455-463. doi: $10.2514 / 3.12008$

[7] Klein, S., and Kok, J., "Sound Generation by Turbulent Non-Premixed Flames," Combustion Science and Technology, Vol. 149, No. 1, 1999, pp. 267-295. doi: $10.1080 / 00102209908952109$

[8] Hirsch, C., Wäsle, J., Winkler, A., and Sattelmayer, T., "A Spectral Model for the Sound Pressure from Turbulent Premixed Combustion," Proceedings of the Combustion Institute, Vol. 31, Elsevier, Amsterdam, 2007, pp. 1435-1441. doi:10.1016/j.proci.2006.07.154

[9] Kraichnan, R., "Diffusion by a Random Velocity Field," Physics of Fluids, Vol. 13, 1970, pp. 22-31. doi:10.1063/1.1692799

[10] Bailly, C., Lafon, P., and Candel, S., "Computation of Noise Generation and Propagation for Free and Confined Turbulent Flows," AIAA Paper 1996-1732, Reston, VA, 1996.

[11] Bailly, C., and Juve, D., "A Stochastic Approach to Compute Subsonic Noise Using Linearized Euler's Equations,” AIAA Paper 1999-1872, Reston, VA, 1999.

[12] Billson, M., Eriksson, L.-E., and Davidson, L., "Jet Noise Prediction Using Stochastic Turbulence Modeling," AIAA Paper 2003-3282, Reston, VA, 2003.

[13] Kalitzin, G., Kalitzin, N., and Wilde, A., "A Factorization Scheme for RANS Turbulence Models and SNGR Predictions of Trailing Edge Noise," AIAA Paper 2000-1982, Reston, VA, 2000.

[14] Ewert, R., and Edmunds, R., "CAA Slat Noise Studies Applying Stochastic Sound Sources Based on Solenoidal Digital Filters," AIAA Paper 2005-2862, Reston, VA, 2005.

[15] Ewert, R., "SLAT Noise Trend Predictions Using CAA with Stochastic Sources from a Random Particle Mesh Method (RPM)," AIAA Paper 2006-2667, Reston, VA, 2006.

[16] Ewert, R., "Broadband Slat Noise Prediction Based on CAA and Stochasic Sound Sources from a Fast Random Particle-Mesh (RPM) Method," Computers and Fluids Journal, Vol. 37, , 2008, pp. 369-387. doi:10.1016/j.compfluid.2007.02.003

[17] Ewert, R., "RPM: The fast Random Particle-Mesh Method to Realize Unsteady Turbulent Sound sources and Velocity Fields for CAA Applications," AIAA Paper 2007-3506, Reston, VA, 2007.

[18] Mühlbauer, B., Noll, B., Ewert, R., Kornow, O., and Aigner, M., "Numerical RANS/URANS Simulation of Combustion Noise," Combustion Noise, edited by A. Schwarz, and J. Janicka, 1st ed., Vol. W, Springer-Verlag, New York, 2009, pp. 1-28.

[19] Ewert, R., and Schröder, W., "Acoustic Perturbation Equations Based on OW Decomposition via Source Filtering," Journal of Computational Physics, Vol. 188, No. 2, 2003, pp. 365-398. doi:10.1016/S0021-9991(03)00168-2

[20] Tam, C., and Auriault, L., "Jet Mixing Noise from Fine-Scale Turbulence," AIAA Journal, Vol. 37, No. 2, 1999, pp. 145-153. doi: $10.2514 / 2.691$

[21] Barlow, R., "Proceedings of the TNF Workshops," Sandia National Lab., Livermore, CA, 1996-2004.

[22] Pope, S., Turbulent Flows, Cambridge Univ. Press, Cambridge, England, 2000.

[23] Mühlbauer, B., Ewert, R., Kornow, O., Noll, B., Delfs, J., and Aigner, M., "Simulation of Combustion Noise Using CAA with Stochastic Sound Sources from RANS," AIAA Paper 2008-2944, Reston, VA, 2008.

[24] Garca-Ojalvo, J., and Sancho, J., Noise in Spatially Extended Systems, Springer-Verlag, New York, 1999.

[25] Brinkmann, B., "Numerische Simulation des Hochfrequenten Effekts von Düsenrandmodifikationen," Masters Thesis, Abteilung Technische Akustik, Institut für Aerodynamik und Strömungstechnik, Deutsches Zentrum für Luft und Raumfahrt, e. V. (DLR), 2007.

[26] Ewert, R., "CAA Prediction of Broadband Trailing-Edge and Jet Noise, in Aeroacoustics Research in Europe: The CEAS-ASC Report on 2007 Highlights," Journal of Sound and Vibration, Vol. 318, Nos. 4-5, 2008, pp. 625-654. doi:10.1016/j.jsv.2008.07.020

[27] Candel, S., Durox, D., Ducruix, S., Birbaud, A.-L., Noiray, N., and Schuller, T., "Flame Dynamics and Combustion Noise: Progress and Challenges," International Journal of Aeroacoustics, Vol. 8, No. 1, 2009, pp. $1-56$. doi:10.1260/147547209786234984

[28] Reynolds, O., "On the Dynamical Theory of Incompressible Viscous UIDS and the Determination of the Criterion," Philosophical Transactions of the Royal Society of London, Series A: Mathematical and Physical Sciences, Vol. A186, 1895, pp. 123-164.

[29] Favre, A., "Equations des Gaz Turbulente Compressible. I. Formes Generales. ,"Journal de Mechanique, Vol. 4, 1965, pp. 361-390.

[30] Strahle, W., "On Combustion Generated Noise," Journal of Fluid Mechanics, Vol. 49, No. 2, 1971, pp. 399-414. doi: $10.1017 / \mathrm{S} 0022112071002167$

[31] Morris, P., and Boluriaan, S., "The Prediction of Jet Noise from CFD Data," AIAA Paper 2004-2977, Reston, VA, 2004.

[32] Morris, P., and Farassat, F., "Acoustic Analogy and Alternative Theories for Jet Noise Predictions," AIAA Journal, Vol. 40, No. 4, 2002, pp. 671680 .

doi:10.2514/2.1699

[33] Gerlinger, P., Numerische Verbrennungssimulation: Effiziente Numerische Simulation Turbulenter Verbrennung, Springer-Verlag, Berlin, 2005. 
[34] Gerlinger, P., Schneider, F., and Aigner, M., "Multivariate Assumed PDF Modeling of Turbulent Sub- and Supersonic Combustion," AIAA Paper 2007-1315, Reston, VA, 2007.

[35] Bui, T., Ihme, M., Meinke, M., Schröder, W., and Pitsch, H., "Numerical Investigation of Combustion Noise and Sound Source Mechanisms in a Non-Premixed AME Using LES and APE-RF," AIAA Paper 2007-3406, Reston, VA, 2007.

[36] Bergmann, V., Meier, W., Wolff, D., and Stricker, W., "Application of Spontaneous Raman and Rayleigh Scattering and 2D LIF for the Characterization of a Turbulent CH4/H2/N2 Jet Diffusion Flame," Applied Physics, Vol. 66, No. 4, 1998, pp. 489-502. doi:10.1007/s003400050424

[37] Meier, W., Barlow, R., and Chen, Y.-L., "Raman/Rayleigh/LIF Measurements in a Turbulent $\mathrm{CH} 4 / \mathrm{H} 2 / \mathrm{N} 2$ Jet Diffusion Flame: Experimental Techniques and Turbulence-Chemistry Interaction," Combustion and Flame, Vol. 123, No. 3, 2000, pp. 326-343. doi:10.1016/S0010-2180(00)00171-1

[38] Schneider, C., Dreizler, A., and Janicka, J., "Flow Field Measurements of Stable and Locally Extinguishing Hydrocarbonfuelled Jet Flames," Combustion and Flame, Vol. 135, Nos. 1-2, 2003, pp. 185-190. doi:10.1016/S0010-2180(03)00150-0

[39] Singh, K., Frankel, S., and Gore, J., "Study of Spectral Noise Emissions from Standard Turbulent Nonpremixed Flames," AIAA Journal, Vol. 42, No. 5, 2004, pp. 931-936. doi: $10.2514 / 1.3424$

[40] Wilcox, D., Turbulence Modeling for CFD, DCW Industries Inc., La Cañada, CA, 2006.

[41] Peters, N., Turbulent Combustion: Cambridge Monographs on Mechanics, Cambridge Univ. Press, Cambridge, MA, 2000.

[42] Zimont, V., "Gas Premixed Combustion at High Turbulence. Turbulent Flame Closure Combustion Model," Experimental Thermal and Fluid Science, Vol. 21, Nos. 1-3, 2000, pp. 179-186.
doi:10.1016/S0894-1777(99)00069-2

[43] Delfs, J., Bauer, M., Ewert, R., Grogger, H., Lummer, M., and Lauke, T., Numerical Simulation of Aerodynamic Noise with DLR's Aeroacoustic Code PIANO: PIANO Manual Version 5.2, Braunschweig, Germany, 2007.

[44] Tam, C., and Webb, J., "Dispersion-Relation-Preserving Finite Difference Schemes for Computational Acoustics," Journal of Computational Physics, Vol. 107, No. 2, 1993, pp. 262-281. doi: $10.1006 /$ jcph. 1993.1142

[45] $\mathrm{Hu}, \overline{\mathrm{F}}$., Hussaini, M., and Manthey, J., "Low-Dissipation and LowDispersion Runge-Kutta Schemes for Computational Acoustics," Journal of Computational Physics, Vol. 124, No. 1, 1996, pp. 177-191. doi:10.1006/jcph.1996.0052

[46] Bilger, R. W., "The Structure of Turbulent Non-Premixed Flames," Twenty-Second International Symposium on Combustion, The Combustion Inst., Pittsburgh, PA, 1988, pp. 475-488.

[47] Georgiadis, N., Yoder, D., and Engblom, W., "Evaluation of TwoEquation Turbulence Models for Jet OW Prediction," AIAA Journal, Vol. 44, No. 12, 2006, pp. 3107-3114. doi: $10.2514 / 1.22650$

[48] Flemming, F., "On the Simulation of Noise Emissions by Turbulent Non-Premixed Flames," Ph.D. Thesis, Technische Universitt Darmstadt, 2006.

[49] Tam, C., Pastouchenko, N., Mendoza, J., and Brown, D., "Combustion Noise of Auxiliary Power Units," AIAA Paper 2005-2829, Reston, VA, 2005.

[50] Tam, C., Golebiowski, M., and Seiner, J., "On the Two Components of Turbulent Mixing Noise from Supersonic Jets," AIAA Paper 96-1716, Reston, VA, 1996.

T. Lyrintzis Associate Editor 